\title{
FACILITATED PARTICIPATORY LEARNING AND ACTION GROUPS TO IMPROVE MATERNAL AND NEWBORN HEALTH AT SCALE IN JHARKHAND, EASTERN INDIA
}

DATA ANALYSIS PLAN

\begin{tabular}{|c|c|}
\hline Study Title & $\begin{array}{l}\text { Facilitated Participatory Learning and Action Groups to } \\
\text { improve maternal and newborn health at scale in } \\
\text { Jharkhand, India }\end{array}$ \\
\hline Short Study Name & Facilitated Learning and Action Groups (FLAG) \\
\hline Registration & $\frac{\text { RIDIE-STUDY-ID-595f13a090784 }}{\text { ISRCTN } 99422435}$ \\
\hline Ethical approval & $\begin{array}{l}\text { Ekjut Independent Ethics Committee }\left(19^{\text {th }} \text { August } 2016\right) \\
\text { University College London Research Ethics Committee } \\
\left(29^{\text {th }} \text { November } 2016 \text {, reference } 1881 / 003\right)\end{array}$ \\
\hline Sponsor & University College London \\
\hline $\begin{array}{l}\text { Version of the Document, reasd } \\
\text { for changes made from ear } \\
\text { version, interim or final version }\end{array}$ & Version 1.5 \\
\hline Date of the current version & $20^{\text {th }}$ January 2020 \\
\hline Authors & $\begin{array}{l}\text { Audrey Prost, Andrew Copas, Hemanta Pradhan, } \\
\text { Tanja A.J. Houweling }\end{array}$ \\
\hline
\end{tabular}




\section{TABLE OF CONTENTS}

1. Study objectives and research questions 3

2. Study design 3

3. Outcome measures 6

$\begin{array}{lll}\text { 4. Sample size calculation } & 7\end{array}$

$\begin{array}{lll}\text { 5. Interim analyses } & 7\end{array}$

6. Approach to final analysis

$\begin{array}{ll}\text { i. general principles } & 7\end{array}$

$\begin{array}{ll}\text { ii. blinding } & 7\end{array}$

$\begin{array}{ll}\text { iii. adjustment } & 8\end{array}$

$\begin{array}{ll}\text { iv. subgroup analysis } & 8\end{array}$

$\begin{array}{ll}\text { v. tables and figures for primary publication } & 8\end{array}$ 


\section{Study objectives and research questions}

The goal of our study is to evaluate the effectiveness, at scale, of women's groups taking part in Participatory Learning and Action (PLA) meetings to improve maternal and newborn health across the state of Jharkhand, in eastern India. These women's group meetings are facilitated by local, government-incentivised community health workers called Accredited Social Health Activists (ASHAs) and their supervisors.

\section{Research questions}

Our impact evaluation seeks to answer two main questions:

1. What is the impact of the intervention on neonatal mortality and maternal and newborn health practices?

2. What is the impact of the intervention on neonatal mortality and maternal and newborn health practices among the socioeconomically most deprived households?

In addition, our process evaluation will answer four questions about coverage, mechanisms, and the role of contextual factors both for the total population and for the socioeconomically most deprived households:

1. What coverage did the intervention achieve?

2. What were the effects of the intervention on women's knowledge related to danger signs in the perinatal period?

3. What were the effects of the intervention on women's decision-making power, selfefficacy and social support during the perinatal period?

4. What factors enabled and hindered impact and scale up?

The full set of indicators and data collection methods to answer these questions is detailed in 3.

\section{Study design}

The intervention's impact will be assessed using a non-randomised, prospective controlled study in six districts of Jharkhand (Latehar, West Singhbhum, Ranchi, Godda, Hazaribagh and Khunti). Jharkhand's State Health Mission and Ekjut, the civil society organisation leading the evaluation, have purposively selected 20 blocks in these six districts. In each of these 20 blocks, they have also selected five data collection clusters of around 10,000 population, or a total of 100 clusters.

Three districts (10 block, 50 clusters) began to receive the intervention in March 2017, while the remaining three districts (10 block, 50 clusters) will receive the intervention in March 2019. The allocation of the early and delayed arms has been decided purposively by the State Health Mission and is therefore non-random. The delayed intervention districts will serve as 'controls' during the evaluation period (2017-2019).

The study design is described in Figure 1.

Figure 1: Study design 


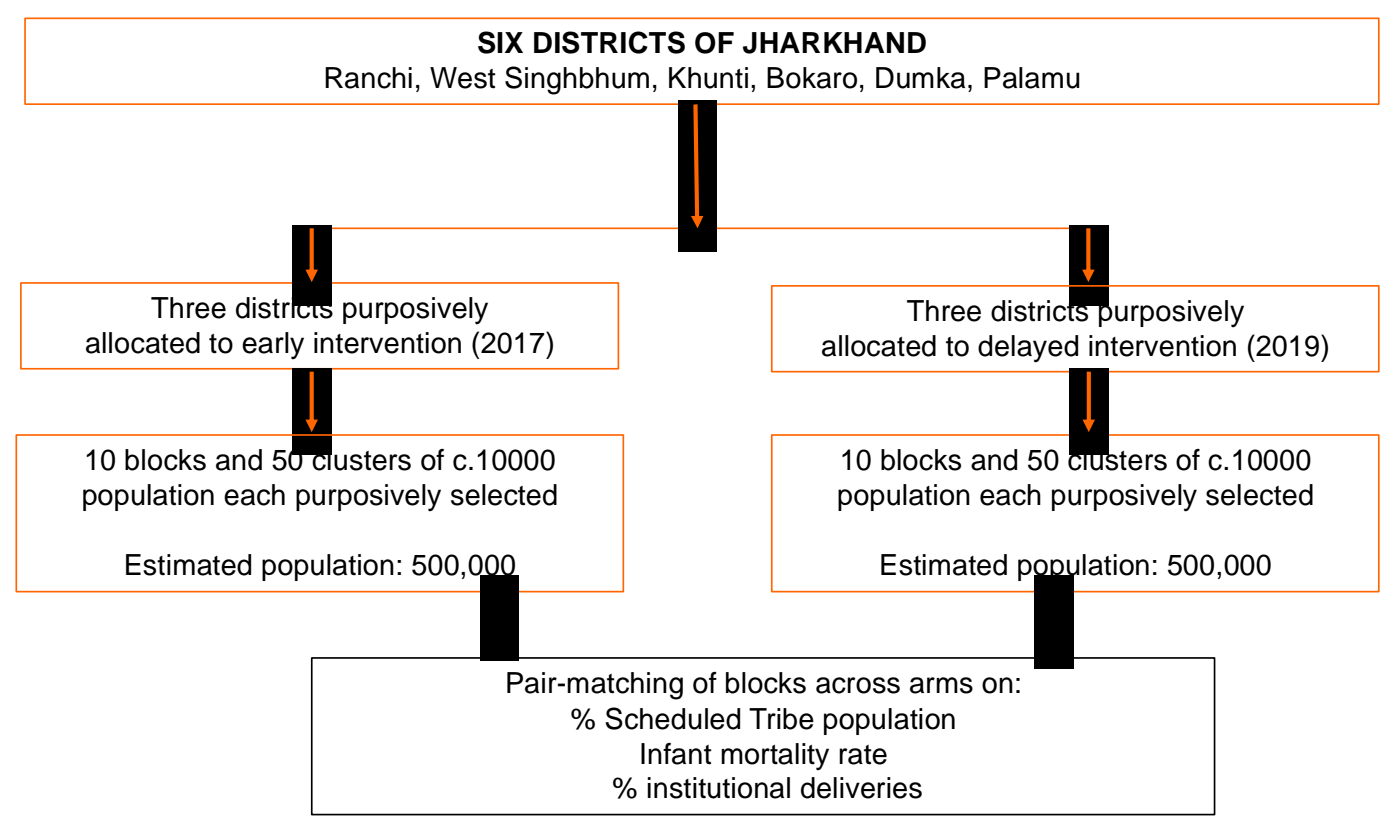

We will collect quantitative data in our 100 surveillance clusters from $1^{\text {st }}$ March 2017 until $31^{\text {st }}$ August 2019 (30 months). The first six months of data collection $\left(1^{\text {st }}\right.$ March $2017-31^{\text {st }}$ August 2017) corresponds to the intervention's start-up time - during which we expect no or only small effects - and is therefore designated as the baseline period. The subsequent 24 months will constitute the evaluation period.

\section{Unit of allocation:}

The unit of allocation is a district (c.100,000 population). The study is a non-randomised prospective, controlled evaluation. Three early intervention districts begin to receive the intervention in 2017, and three delayed intervention districts receive it in 2019.

\section{Early and delayed exposures:}

In three early intervention districts, State Health Mission master trainers trained district and blocklevel teams in early 2017. These teams have trained ASHA supervisors in those ten blocks to conduct PLA meetings in half of their catchment areas, and provide on the job training to ASHAs to conduct meetings in the other half of their catchment area. The participatory learning and action meetings follow a cycle in which groups: (1) identify and prioritise common problems faced by women and children during pregnancy, delivery, and the postnatal period; (2) discuss the causes and consequences of these problems, and identify strategies to address them; (3) implement their strategies; (4) assess the meeting cycle.

In the three delayed intervention districts, the training of ASHA supervisors and ASHAs will begin in early 2019.

\section{Participants, eligibility and migration:}


Two types of respondents are eligible to participate in the questionnaire survey that will be administered in our 100 data collection clusters:

1. Women aged 15 and above residing in the clusters and who delivered a baby, or experienced a stillbirth or neonatal death between $1^{\text {st }}$ March 2017 and $31^{\text {st }}$ August 2019

2. Relatives of women who have died as a result of complications of pregnancy and childbirth (maternal deaths).

We will exclude women who have migrated for three months or more since their identification.

\section{Sample size calculation}

We estimated that there would be a minimum of 200 live births per cluster of 10000 population per year, or 400 live births over the evaluation period of two years, i.e. 2000 births per block with five data collection clusters. We estimated the current state-level NMR at 35 per 1000 livebirths, allowing for inter- and intra-district variations. We initially estimated that an evaluation with a total of 10 blocks (50 clusters) beginning the women's group intervention in 2017, and 10 blocks of 50 clusters beginning the intervention in 2019 would be able to detect a reduction of $20 \%$ in neonatal mortality between these two groups of blocks between $1^{\text {st }}$ October 2017 and $31^{\text {st }}$ September 2019 using a known $\mathrm{k}$ (coefficient of variation between clusters) of 0.1 (from published data), from 35 per 1000 livebirths to 28 per 1000 livebirths with $80 \%$ power at the $95 \%$ significance level. The evaluation would also be powered to detect a $25 \%$ reduction in NMR among the $40 \%$ most deprived, from an estimated 40 per 1000 live births to 30 per 1000 live births. A few months after the start of the evaluation, the allocation changed from block-level to district-level, with substantial implications for the power of the evaluation. We did not recalculate sample size following this change and maintained the original evaluation design.

We estimate that interviewers will capture information for around 50,000 births $(10,000$ during the baseline and 40,000 during the evaluation) over 30 months across 100 clusters. These data will allow us to robustly estimate the impact of the scaled-up intervention on neonatal mortality, and on maternal and newborn health practices.

\section{Outcome data collection}

In each of the 100 data collection clusters, a network of five to six key informants (lay female community members) will report births or deaths to girls and women aged 15 and above to an interviewer employed by the evaluation team. One interviewer will be responsible for a cluster of 10,000 population and conduct $1-2$ interviews per day. They will visit key informants on a monthly basis to collect a list of births and deaths, verify the accuracy of these reports, pay the key informant an incentive of INR 50 (GBP 0.5) for each correct identification, and visit each household where a birth or death has been identified. During this visit, which will take place between six and eight weeks after the identified event, the interviewer will seek informed consent from mothers (or, in the event of a maternal death, other family members), and conduct an interview about practices in pregnancy, delivery and the postnatal period.

Interview data will be collected on a smartphone using an application developed in CommCare.

Additional qualitative data are collected for process evaluation, but these data are not considered within this analysis plan.

\section{Outcome measures (last revised $5^{\text {th }}$ July 2017)}

\subsection{Outcomes for the impact evaluation}

\section{Primary outcome}


Neonatal mortality measured via a questionnaire survey for all women who gave birth in the study areas during the study period (1st March 2017-30th August 2017 for the baseline period, and 1st September 2017-30th August 2019 for the evaluation period)

\section{Secondary outcomes}

All secondary outcomes are measured via a questionnaire survey for all women who gave birth in the study areas between 1st March 2017 and 30th August 2019.

They include:

1. Stillbirth rate (per 1000 births)

2. Perinatal deaths (per 1000 births)

3. Neonatal mortality among mothers in the most socio-economically deprived, defined as

mothers belonging to the two poorest wealth quintiles and who cannot read

4. Maternal deaths

5 , \% Mothers who received three ANC consultations by a skilled provider

6. \% Mothers who made plans for birth (transport, location, money) in pregnancy

7. \% Mothers who sought skilled care for a problem in pregnancy

8. \% Births with a skilled attendant

9. \% Births in a health facility

10. \% Home births where a clean delivery kit was used

$11 . \%$ Infants wiped within 30 mins of birth

12. \% Infants wrapped within 30 mins of birth

13. \% Infants not given a bath in 1 st 24 hours

14. \% Infants given breastmilk within one hour

15. \% Infants exclusively breastfed

16. \% Mothers visited by ASHA three times in the first week

17. \% Infants for whom skilled care is sought for a newborn health problems

18. \% Mothers who receive a postpartum check-up from a skilled provider

\subsection{Outcomes for the process evaluation}

Our questionnaire survey will also capture outcomes for the process evaluation.

Specifically, we will report, for both the total population and the socioeconomically most deprived:

\section{Coverage}

$\%$ of women who have recently delivered who reported ever participating in a PLA meeting

\section{Knowledge of danger signs}

Mean number of dangers signs in pregnancy, after birth and for newborn infants known by women who recently delivered

Decision-making power, self-efficacy and social support

- \% of mothers who report taking decisions about their routine healthcare during last pregnancy by themselves, jointly with their husbands, or jointly with other family members

- \% of mothers who report feeling very confident or confident that they knew how to look after themselves and their baby during pregnancy, the delivery and the newborn period

$\%$ of mothers who reported feeling supported by their community to solve problems related to pregnancy, delivery and newborn health. 


\section{Interim analyses}

We do not expect any adverse effects of the intervention but plan to carry out two interim analyses (in 2017 and 2018) prior to the final analysis in 2019, as part of the Annual Programme Review conducted with the Children's Investment Fund Foundation.

In November 2017, we will review data for all primary outcomes (neonatal mortality, maternal deaths), secondary outcomes (preventive and care-seeking behaviours), and quantitative process evaluation outcomes for the baseline period ( $1^{\text {st }}$ March 2017-31 ${ }^{\text {st }}$ August 2017 . We will present these for the total population, for the most socioeconomically disadvantaged, and by district.

In 2018, we will review data for three selected 'tracer' outcomes in 3 early vs 3 delayed districts for the purpose of programme review:

- \% mothers who made plans for birth (transport, location, money) in pregnancy

- $\%$ of infants given breastmilk within one hour of birth

- \% Infants wrapped within 1 hour of birth

\section{Approach to final analysis}

i. General principles and adjustment

Analyses will be by intention-to-treat, meaning they are based on eligible mothers and infants analysed according to allocation, regardless of whether they received the intended exposure, i.e. regardless of whether or not they attended women's group meetings.

The focus of analysis is measuring the difference between early and delayed allocation arms, leading to odds ratios for binary outcomes and mean differences for continuous outcomes.

As this evaluation compares data from three early intervention districts to three delayed intervention districts, it is akin to the analysis of a trial with a small number of clusters. Following the principles described in Hayes \& Moulton (2017), we will conduct a cluster-level analysis, with districts being treated as clusters. Our primary outcome is binary, hence the cluster summary values are proportions. We will analyse these 'raw' proportions but our primary analysis will be on the adjusted cluster summary values. As described in Hayes \& Moulton (2017) the adjusted values are derived by first predicting the expected number of outcome events (deaths) for each cluster (in the absence of an intervention effect) from fitting a logistic regression model for each outcome based on variables defined a priori on the basis of either expected strong prediction or difference observed at baseline: tribe/caste, maternal schooling, maternal literacy and asset quintile. The process is conducted separately for baseline and evaluation period for each cluster. The final analysis of the intervention effect is an ANCOVA analysis i.e. a linear regression of cluster summary values from the evaluation period adjusted for the summary values at baseline, including allocation (early vs. late implementation) as a covariate.

\section{ii. Blinding}

The data analysts (HP and AP) will conduct the baseline analysis blinded to allocation in November 2017. The final analysis (2019) will also be conducted by the analysts (HP and AP) blinded, preparing effect measures that compare "Arm A" with "Arm B". The report, do files, and a dataset with arms identified as "Arm A" and "Arm B" will then be handed over for checking by CIFF staff, and made available to members of the Independent Advisory Committee. An independent statistician will check the STATA do file.

We will present all outcomes with and without adjustment for values at baseline, and consider 
adjusted measures as the main measures.

iii. Subgroup analyses

We will collect data on the socio-demographic characteristics of mothers and use them to determine the impact of the intervention among the socioeconomically most deprived mothers and children. We will examine effects for 15 outcomes (neonatal mortality and all preventive and care-seeking practices). To do this, we will repeat the primary analysis, restricted to mothers and children at the (approximate) bottom $40 \%$ of the socioeconomic hierarchy, defined on the basis of household ownership of assets (belonging to the two poorest quintiles) and literacy (cannot read or only with difficulty).

We plan to carry out a further, descriptive subgroup analysis in addition to the analyses for the most socioeconomically deprived. The subgroup will be defined by intensity of mothers' exposure to group meetings (number of meetings attended, grouped into 3 categories: none, less than $10,10+)$. The subgroup analysis will be based initially on the primary outcome. We will present descriptive mortality rates descriptively based for each group, without formal testing.

iv. Tables and figures for primary publication

We will include a table to describe the 'baseline' characteristics of mothers and infants by allocation arm, based on percentages or means or other summary statistics. These characteristics will be district, maternal education, literacy, tribal/caste status, and asset quintile. We will then for each outcome listed in section 3 present in one or more tables the appropriate summary statistics for each arm based on available cases, and then the appropriate effect measure with and without adjustment and in each case with a $95 \%$ confidence interval. We will present data both for the total population and for the most socioeconomically disadvantaged. We will include a diagram to show rates of data collection in each arm along with refusal and migration, and deaths (stillbirths, neonatal deaths, and maternal deaths). For neonatal mortality, we will present a figure to illustrate changes by arm, over time. 